\title{
Diagnostic Utility of Interferon-Gamma Release Assay in
}

\section{Tuberculous Lymphadenitis}

\author{
Xinchao Liu ${ }^{1 \dagger}$, Susu Ye ${ }^{1 \dagger}$, Wenze Wang ${ }^{2}$, Yueqiu Zhang ${ }^{1}$, Lifan Zhang ${ }^{1,3}$, Xiaocheng Pan ${ }^{4}$, Ziyue Zhou ${ }^{4}$, \\ Miaoyan Zhang ${ }^{4}$, Jianghao Liu ${ }^{4}$, Zhiyong Liang ${ }^{2}$, Xiaoqing Liu ${ }^{1,3^{*}}$ \\ 1 Department of Infectious Diseases, Peking Union Medical College Hospital \\ 2 Department of pathology, Peking Union Medical College Hospital \\ 3 Clinical Epidemiology Unit, International Epidemiology Network, \\ 4. Department of Internal Medicine, Peking Union Medical College Hospital \\ Chinese Academy of Medical Sciences \& Peking Union Medical College, Beijing 100730, China
}

Key words: Tuberculous lymphadenitis (TBL); T-SPOT.TB; diagnostic test; sensitivity; specificity

Objective The aim of this study was to evaluate the diagnostic performance of T-SPOT.TB for tuberculous lymphadenitis.

Methods Suspected tuberculous lymphadenitis patients between September 2010 and September 2018 who had both peripheral blood T-SPOT.TB test and lymph node biopsy were retrospective enrolled in this study. The cutoff value of TSPOT.TB test for peripheral blood was set as $24 \mathrm{SFC} / 10^{6} \mathrm{PBMC}$ according to the testing kits. The gold standard for diagnosis of TBL is combination of tissue culture or acid-fast staining, or histological findings consistent with TBL and clinical or radiological response to anti-TB treatment. Diagnostic efficacy of T-SPOT.TB was evaluated, including sensitivity, specificity, accuracy, predictive values, and likelihood ratio.

Results Among 91 patients who met the inclusion criteria, we excluded 8 cases with incomplete clinical information and 6 cases who lost of follow-up. According to the gold standard, there were 37 cases of true TBL (9 confirmed TBL and 28 probable TBL), 30 cases of true non-TBL, and 10 patients of clinically indeterminate diagnosis which were excluded from the final analysis. T-SPOT.TB tests yielded 43 cases of positive response and 24 cases of negative response by. The sensitivity, specificity, accuracy, positive predictive value (PPV), negative predictive value (NPV), positive likelihood ratio (PLR) and negative likelihood ratio (NLR) of peripheral blood T-SPOT.TB for diagnosing TBL were 89.2\%, 66.7\%, 79.1\%, 76.7\%, 83.3\%, 2.676 and 0.162, respectively. The number of spot forming cells (SFCs) of T-SPOT.TB were [432(134-1264)/106 PBMCs] in TBL patients, higher than that in non-TBL patients $\left[0(0-30) / 10^{6} \mathrm{PBMCs}\right]$ with significant statistical difference $(\mathrm{Z}=-5.306, P<0.001)$.

Conclusions T- SPOT.TB is a rapid and simple diagnostic test for TBL with a high sensitivity and negative predictive value.

Received August 16, 2018; accepted March 22, 2019; published online November 12, 2019.

* Corresponding author phone 010-69155087; E-mail: liuxq@pumch.cn

+ These authors contribute equally

Grant supported: Ministry of Science and Technology of the People's Republic of China (2014ZX10003003); Chinese Academy of Medical Sciences Initiative for Innovative Medicine(2016-12M-1-013), and Chinese Academy of Medical Sciences Fund for Tuberculosis Research ( 2016ZX310183-4) 
Tuberculosis(TB), an infectious disease and serious health hazard, caused 1.3 million deaths among HIV-negative people in 2017 according to the World Health Organization (WHO). ${ }^{1}$ China bears a high burden of TB in the world. According to the estimation of WHO, in 2016 China had 895000 new infections of TB, which ranked only after India and Indonesia. Tuberculous lymphadenitis (TBL) is the most common extra-pulmonary tuberculosis, which accounts for $4.0 \%$ to $5.1 \%$ of all tuberculosis and $14.5 \%$ to $50.0 \%$ of extra-pulmonary tuberculosis cases. ${ }^{2-5}$ The clinical manifestations of TBL can be atypical, and misdiagnosis is common. It usually requires tissue culture and pathological verification to make a definitive diagnosis. However, the cultivate yield from lymph node biopsy is usually extremely low, therefore it is paramount to develop a rapid, sensitive and less invasive diagnostic utility.

Interferon gamma release assay (IGRA), including QuantiFERON-TB Gold and T-SPOT.TB, is based on cellular immune response, and has been introduced into the diagnosis of tuberculosis in recent years. It was reported that IGRA used in the diagnosis of TBL had sensitivities of $78.8 \%-95.2 \%$ and specificities of $52 \%-95.5 \%$. ${ }^{4,6-13}$. The great varieties of these results are likely due to the inability to acquire both IGRA and lymph node biopsy simultaneously, and the small sample size in each study. Some studies were designed for all entities of extra-pulmonary tuberculosis rather than TBL specifically, making them less specific for TBL evaluation. $4,6,12,13$ This study was designed to explore the diagnostic utility of T-SPOT.TB in TBL by using results from tissue culture, pathology and clinical follow-up.

\section{MATERIALS AND METHODS}

\section{Patients selection}

This retrospective study was reviewed and approved by the Institutional Review Board of the hospital and patient information consent was waived. The study was designed to evaluate suspected TBL patients from Peking Union Medical College Hospital between September 2010 and September 2018. We retrospective searched databases to retrieve all inand outpatients with both results of lymph node biopsy pathology and peripheral blood T-SPOT.TB. Histological diagnosis was obtained for the lymphadenopathy from all cases either by thoracic/abdominal surgery or by superficial lymph node biopsy. Clinical information including demographic characteristics, symptoms, underline conditions, sites of biopsy etc, was acquired through charter review. The inclusion criteria for patients are as follows: 1 . Age greater than 18 years old; 2 . at least one of the following manifestations: (1) symptoms of fever, weight loss, or night sweat; (2) peripheral lymph node swelling by physical examination; (3) lymph node enlargement confirmed by CT or MRI. Cases with insufficient charter information to make definitive diagnosis or lost follow up were excluded.

Two physicians specialized in infectious disease made categorization of patients' diagnosis independently based on patients' clinical manifestations, imaging findings, microbiology, pathology results, and therapeutic effect of antituberculosis treatment in the follow up period without knowing T-SPOT.TB results. For cases whose definitive diagnosis could not be reached from the initial visit, we further reviewed medical records of the following three months, or we followed up by phone call to determine anti-TB treatment response of the patient. When the two physicians perceived different opinions, a third specialist would be consulted for the final diagnosis.

\section{Diagnostic categorization for TBL}


The gold standard for TBL was the combination of microbiology result, pathology result, and patients' response to antituberculosis treatment. Patients were categorized into 4 groups according to protocols in the study of Liao $\mathrm{CH}$ et al. ${ }^{3}$ Confirmed TBL and probable TBL was considered as true TBL according to the gold standard.

1. Confirmed TBL: Mycobacterium tuberculosis (MTB) culturing of lymph node tissue is positive, or tissue Acidfast staining is positive.

2. Probable TBL: histological findings are consistent with TBL (granulomatous inflammation and/or caseous necrosis and/or multinucleate giant cell), and the patient responds clinically and radiologically to anti-TB treatment.

3. Indeterminate TBL: do not meet the above diagnostic criteria or fail to reach a conclusive diagnosis during a 3-months follow-up.

4. Non-TB: alternative diagnosis is made, or there is clinical improvement without anti-TB therapy.

\section{T-SPOT.TB test}

T-SPOT.TB is generally ordered for patients who frequently present with fever, weight loss, night sweat, and elevated erythrocyte sedimentation rate (ESR), suspecting for TB infection. We collected $4 \mathrm{ml}$ of heparin-anticoagulated venous blood from these patients, and blood samples were sent for T-SPOT.TB (Oxford Immunotec, Abingdon, UK) test within 6 hours by a laboratory personnel who was blinded to patients' clinical data. T-SPOT.TB utilized AIM-V (GIBCOTM AIM V Medium liquid, Invitrogen, US.) as negative control, phytohemagglutinin (PHA) as positive control, early secreted antigenic target 6-kDa protein (ESAT-6) and culture filtrate protein 10 (CFP-10) as specific antigens, respectively. Peripheral blood monocyte cells (PBMC) from each subject were separated and prepared at a concentration of $2.5 \times 10^{6} / \mathrm{ml}$, and then plated $\left(2.5 \times 10^{5}\right.$ per well $)$ on a plate precoated with antibody against interferon $\mathrm{Y}$. Plates were incubated $16-18 \mathrm{~h}$ at $37^{\circ} \mathrm{C}$ in $5 \%$ carbon dioxide. After incubation, wells were developed with a conjugate against the antibody used and an enzyme substrate. Spot-forming cells (SFCs) were counted with an automated ELISPOT reader (AID-ispot, Strassberg, Germany), with each SFC representing an antigen-specific T cell secreting interferon $Y$. The response was considered positive if the antigen well contained 6 or more spots and twice the number of spots as seen in the negative control well based on the T-SPOT.TB kit (Oxford Immunotec, Abingdon, UK) operating instruction. The background spots number should be less than 10 of the negative control well for PBMC and greater than 20 of the positive control well for PBMC. The cutoff value of T-SPOT.TB test for peripheral blood was set as $24 \mathrm{SFC} / 10^{6} \mathrm{PBMC}$ according to the testing kits.

\section{Statistical analysis}

Statistical analysis was performed using SPSS 19.0 software (SPSS Inc, Chicago, IL). Normal distribution was determined by Kolmogorov-Smirnov tests. Continuous data of normal distribution was expressed as mean \pm standard deviation, while non-normal distributional data was expressed as medians and interquartile ranges. Classification data was expressed as percentage. T-test and Mann-Whitney $\mathrm{U}$ test were used to compare continuous variables of normal distribution and non-normal distribution, respectively. The $95 \%$ confidence interval ( $95 \% C I$ ) was estimated from the binomial distribution. $P<0.05$ was considered statistically significant. 


\section{RESULTS}

\section{Characteristics of the subjects}

From patients who had both T-SPOT.TB and lymph node biopsy pathology reports available in our database, 91 patients with suspicious tuberculous lymphadenitis were included in this study. Among them, we excluded 6 cases who lost follow up and 8 cases with incomplete clinical information from analysis. The process of patient selection is illustrated in Figure 1. Of the remaining 77 patients, 28 were male and 49 were female; their age ranged from 18 to 75 years old, with mean of $47.9 \pm 14.1$ years old. The HIV serological antibody tests of all subjects were negative. There were 9 confirmed TBL cases, 28 probable TBL cases, 10 indeterminate TBL cases, and 30 non-TB cases. The clinical demographic characteristics of the enrolled patients are shown in Table 1.

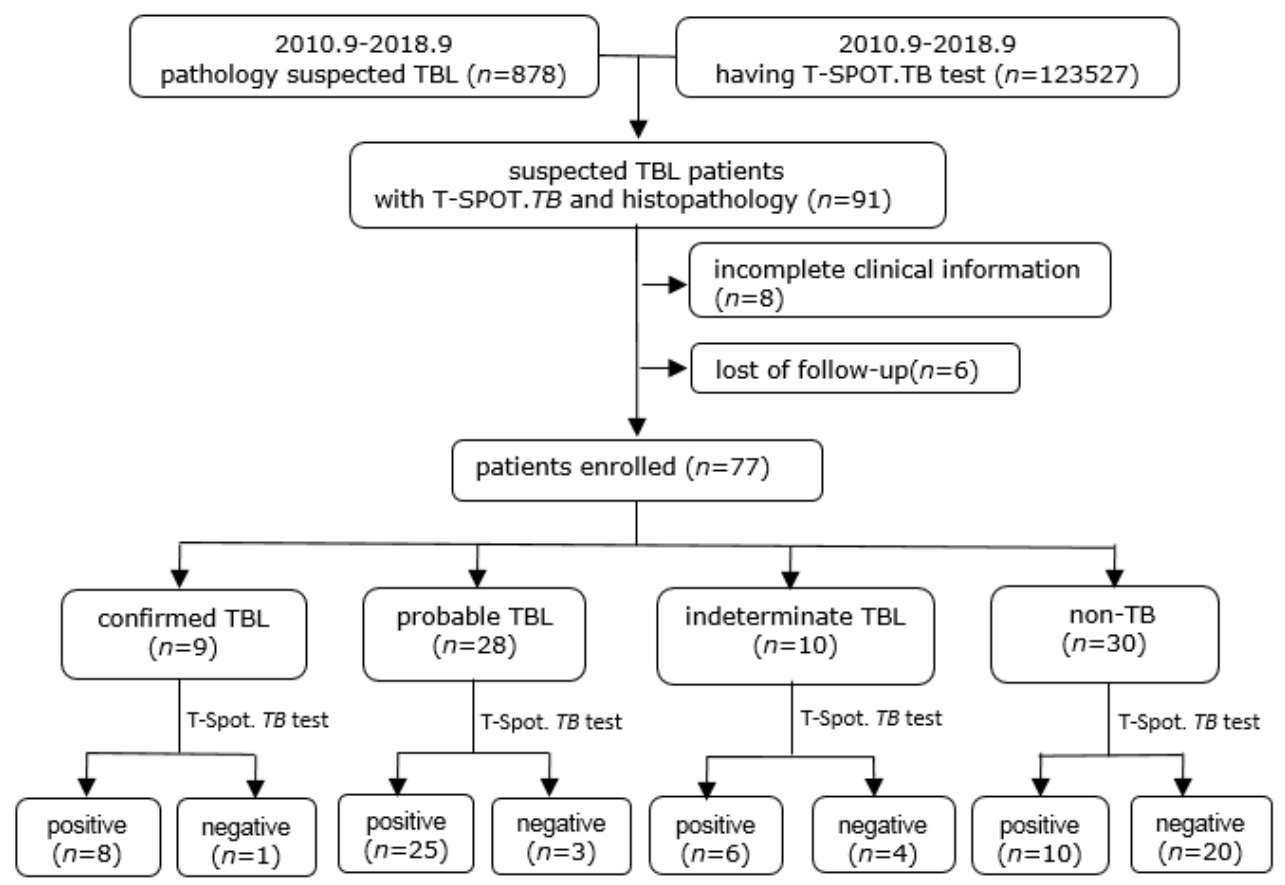

Figure 1. Flowchart of enrolling patients in the study.

Table 1. Demographic and clinical characteristics of suspected TBL patients enrolled $(n=77)$

\begin{tabular}{|c|c|c|c|c|}
\hline Characteristic & $\begin{array}{l}\text { Confirmed TBL } \\
\qquad n=9 \\
\end{array}$ & $\begin{array}{c}\text { Probable TBL } \\
\quad n=28 \\
\end{array}$ & $\begin{array}{l}\text { Indeterminate } \\
\qquad n=10 \\
\end{array}$ & $\begin{array}{c}\text { Non-TBL } \\
n=30 \\
\end{array}$ \\
\hline Age (years, mean $\pm S D$ ) & $43.1 \pm 17.3$ & $46.5 \pm 16.0$ & $49.9 \pm 11.9$ & $50.0 \pm 12.0$ \\
\hline \multicolumn{5}{|l|}{ Gender $(n)$} \\
\hline male & 5 & 7 & 4 & 12 \\
\hline female & 4 & 21 & 6 & 18 \\
\hline TB history positive (n) & 1 & 6 & 0 & 1 \\
\hline \multicolumn{5}{|l|}{ Co-Medication (n) } \\
\hline Steroids & 1 & 5 & 2 & 2 \\
\hline
\end{tabular}




\begin{tabular}{|c|c|c|c|c|}
\hline Immunosuppressive agent* & 1 & 2 & 0 & 1 \\
\hline Underlying condition $(n)$ & $7(77.8 \%)$ & $8(28.6 \%)$ & $3(30 \%)$ & $6(20 \%)$ \\
\hline Cancer & 1 & 1 & 1 & 2 \\
\hline Diabetes mellitus & 2 & 2 & 1 & 2 \\
\hline Chronic liver diseases & 2 & 1 & 1 & 0 \\
\hline Chronic kidney diseases & 1 & 0 & 0 & 1 \\
\hline Autoimmune diseases & 1 & 4 & 0 & 1 \\
\hline \multicolumn{5}{|l|}{ Blood tests } \\
\hline \multicolumn{5}{|l|}{ Leukocyte } \\
\hline$\left(\times 10^{9} / \mathrm{L}\right.$, median IQR) & $7.55(6.04-8.41)$ & $6.09(4.84-7.67)$ & $4.17(3.98-6.23$ & $7.82(4.64-11.78$ \\
\hline Lymphocyte & 1.60 & 1.37 & 0.97 & 1.37 \\
\hline$\left(\times 10^{9} / \mathrm{L}\right.$, median IQR) & $(1.07-3.14)$ & $(1.07-1.78)$ & $(0.77-1.54)$ & $(0.97-1.91)$ \\
\hline Erythrocyte sedimentation rate & $38(13-57)$ & $12(4.51-19.75)$ & $43(23-69)$ & $22(14.25-39.25)$ \\
\hline \multicolumn{5}{|l|}{ C-reactive protein $(\mathrm{mg} / \mathrm{dL}$, } \\
\hline median IQR) & $8.3(4.1-49.0)$ & $5.9(1.7-18.8)$ & $6.1(2.8-10.3)$ & $4.9(2.1-32.4)$ \\
\hline Site of lymph node biopsy ${ }^{\ddagger}(n)$ & 13 & 30 & 14 & 31 \\
\hline Postauricular & 0 & 2 & 0 & 0 \\
\hline Submandibular & 1 & 2 & 1 & 0 \\
\hline Cervical & 2 & 7 & 3 & 8 \\
\hline Supraclavicular & 3 & 5 & 1 & 4 \\
\hline Axillary & 1 & 1 & 1 & 2 \\
\hline Mediastinal & 4 & 8 & 4 & 13 \\
\hline Celiac & 2 & 5 & 4 & 1 \\
\hline Inguinal & 0 & 0 & 0 & 3 \\
\hline \multicolumn{5}{|l|}{ Histopathologic finding $(n)$} \\
\hline Granuloma & 9 & 28 & 10 & 26 \\
\hline Caseous necrosis & 6 & 9 & 0 & 0 \\
\hline Multinucleate giant cell & 4 & 16 & 4 & 6 \\
\hline
\end{tabular}

*After administration of Thalidomide and Cyclosporin A. ¥Certain patient may have more than one lymph node biopsy.

TB, tuberculosis; TBL, tuberculous lymphadenitis; IQR, interquartile range.

\section{The diagnostic performance of T-SPOT.TB for TBL}

Of the enrolled 77 patients, according to the gold standard,37 patients were true positive of TBL ( 9 confirmed TBL cases and 28 probable TBL cases), 30 cases were true negative of TBL. The indeterminate TBL cases $(n=10)$ were excluded from the diagnostic test. Of 37 TBL cases, T-SPOT.TB was positive in 33 cases, with the sensitivity of $89.2 \%$ ( $95 \%$ CI, $73.6 \%-96.5 \%)$. In 30 true non-tuberculosis patients, 20 cases were negative for T-SPOT.TB, with the specificity of $66.7 \%(95 \%$ CI, 47.1\% - 82.1\%) (Table 2). The accuracy of T-SPOT.TB for TBL was 79.1\%. The positive predictive value, negative predictive value, positive likelihood ratio and negative likelihood ratio of T-SPOT.TB for TBL were 76.7\% (95\% CI, 61.0\%-87.7\%), 83.3\% (95\% CI, 61.8\%-94.5\%), 2.68 (95\% CI, 1.59-4.49) and 0.16 (95\% CI, 0.06-0.42), respectively. 
Table2. Diagnostic yields of T-SPOT.TB for the suspected tuberculous lymphadenitis

\begin{tabular}{cccc}
\hline & \multicolumn{2}{c}{ Diagnosed by gold standard } & \multirow{2}{*}{ Total } \\
\cline { 2 - 3 } T-SPOT.TB & TBL & Non-TBL & \\
\hline Positive & 33 & 10 & 43 \\
Negative & 4 & 20 & 24 \\
Total & 37 & 30 & 67 \\
\hline
\end{tabular}

\section{The number of IFN - Y - secreting T cells(SFCs, Spot Forming Cells) in peripheral blood}

The number of ESAT-6 antigens in true TBL group and true non-TBL group were 236 (48-508) SFCs / 106 PBMCs and $0(0-0)$ SFCs $/ 10^{6}$ PBMCs, respectively $(Z=-5.274, P<0.001)$ (Figure 2). The number of CFP-10 antigens in the TBL group and non-TBL group were 148 (36-690) SFCs / $10^{6}$ PBMCs and 0 (0-28) SFCs / $10^{6}$ PBMCs, respectively ( $Z=-$ 4.630, $P<0.001)$. The tuberculosis mycobacteria specific antigen T-SPOT.TB SFCs, which was the sum of ESAT-6 and CFP-10 SFCs, of the TBL group and non-TBL group were 432(134-1264) SFCs / 106 PBMCs and 0 (0-30) SFCs / 106 PBMCs, respectively $(Z=-5.306, P<0.001)$.

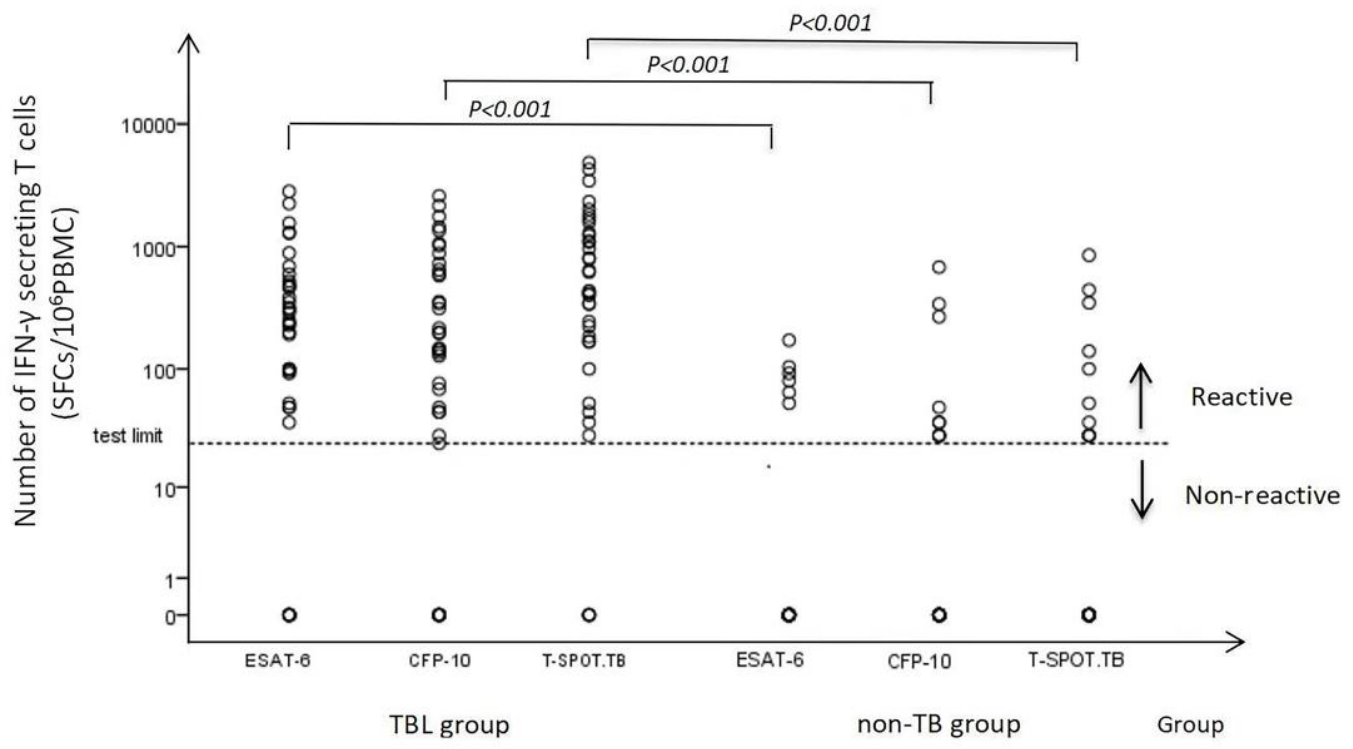

Figure2. The number of mycobacterium tuberculosis specific IFN- $\gamma$ secreting $T$ cells in peripheral blood of TBL patients and Non-TBL patients.

Significant difference in the number of ESAT-6, CFP-10 and total T-SPOT.TB spots between TBL group and the non-TB group (all $\mathrm{P}<0.001$ ). The segmentation line indicates the threshold value of T-SPOT.TB ( $24 \mathrm{SFCS} / 10^{6}$ PBMC). Results below the threshold wereall present as 0 in the final report. ESAT-6, early secreted antigenic target 6-kDa protein; CFP-10, culture filtrate protein 10.

\section{DISCUSSION}

Tuberculosis is an important threatening for public health. In 2017 it caused up to 1.3 million and 3.0 million deaths in HIV negative and positive patients respectively. ${ }^{1}$ Tuberculous lymphadenitis is one of the most common extra pulmonary tuberculosis. The clinical manifestations of tuberculous lymphadenitis are mostly non-specific, which makes the definitive diagnosis quite challenging. At present, the most commonly used test for Mycobacterium tuberculosis 
infection is still the tuberculin skin test. But its low sensitivity and low specificity, ${ }^{14,15}$ potential influenced by bacille Calmette-Guerin (BCG) vaccination and environmental mycobacterial infection, not being applicable in immunosuppressed patients, and severe tuberculosis patients, have limited its universal clinical application.

Ever since Koch discovered Mycobacterium tuberculosis in 1882, acid-fast staining has been the cornerstone of tuberculosis diagnosis. ${ }^{16}$ The number of Mycobacterium tuberculosis in tissue samples needs to be greater than $10^{4} / \mathrm{ml}$ to yield a positive acid-fast staining result. But tuberculous lymphadenitis usually contains very few Mycobacterium tuberculosis, which makes the sensitivity of acid-fast staining in diagnosing TBL extremely low (0-40\%). ${ }^{17,18}$ Also, nontuberculous mycobacterium can be acid-fast positive. We found only 9 out of 37 TBL patients (24.3\%) were positive for acid-fast staining in this study. Mycobacterium tuberculosis culture and identification is still diagnostic gold standard, which usually takes 4-6 weeks to isolate, and the positive rate of culturing can be as low as $30 \%-67.2 \% .{ }^{19}$ Polymerase chain reaction (PCR) can be a useful method in diagnosing Mycobacterium tuberculosis infection, with sensitivities and specificities of $88 \%-90.3 \%$ and $82 \%-100 \%$, respectively, ${ }^{20-22}$ and the result can be obtained in a few hours. However, PCR in diagnosing TBL requires invasive methods for sampling, professional staff and advanced laboratory facilities. Furthermore, it cannot determine whether the Mycobacterium tuberculosis in tissue is alive or not. 23,24

In recent years, IFN-y release assays (IGRA) have been developed for screening tuberculosis infections, including whole blood IFN-y enzyme-linked immunosorbent assay (QuantiFERON-TB Gold) and enzyme-linked immunospot assay (T-SPOT.TB). T-SPOT.TB is a new immunological diagnostic tool for the detection of Mycobacterium tuberculosis infection. It is not influenced by BCG vaccination and most infections of non-tuberculous mycobacteria. The sensitivity of T-SPOT.TB is also high in that one specific T lymphocytes can be detected in every 1 million peripheral blood mononuclear cells and the entire detection process only takes 2 days. ${ }^{25}$ According to the peripheral blood T-SPOT.TB result in current study, the number of spot forming cells was significantly higher in TBL patients than in the non-TBL patients. Peripheral blood T-SPOT.TB showed a good sensitivity $(89.2 \%)$ but a relative low specificity $(66.7 \%)$ for diagnosis of TBL. These results were comparable with that in previous reports where T-SPOT.TB in diagnosing TBL had sensitivities of $81 \%-95 \%$, specificities of $52 \%-80 \% .{ }^{10-13}$ It was reported that false negative T-SPOT.TB results might be associated with elderly age, serious comorbidities such as liver cirrhosis, and other underlying diseases. ${ }^{26,27}$ Thus, TSPOT.TB should be interpreted discreetly in these population. In the current study, 4 cases of 37 TBL patients were TSPOT.TB negative.

We found that peripheral blood T-SPOT.TB had a good negative predictive value (83.3\%) for TBL, which was high, and this result was consistent with the study of Jia et al ${ }^{10}$ who reported a negative predictive value as high as $95.9 \%$. Therefore, T-SPOT.TB may have a great value in excluding the diagnosis of TBL. Of the non-TBL group, 10 patients had positive T-SPOT.TB results (FPR 33.3\%), but only one of them had a history of tuberculosis. We speculate that these cases may have latent tuberculosis infections. Gao and colleagues reported a high prevalence of latent tuberculosis in China, ${ }^{28}$ with the QuantiFERON-TB positive rate being $13 \%-20 \%$, which may explain the relatively low specificity of TSPOT.TB in diagnosing TBL. 
In this study the number of T cells that responded to ESAT- 6 in the TBL group appeared to be higher than the number of T cells that responded to CFP-10, but the difference was not statistically significant. Similar findings were reported in female genital tuberculosis patients ${ }^{29}$ and in active TB infection populations. ${ }^{30}$ However, as these studies are not specifically targeting patients of TBL, more TBL specific researches are needed.

TBL is generally considered to be prevalent mainly in children. However, to our clinical experience in recent year, it is commonly seen in people of 20-40 years old, especially in women. The gender preference was documented in this study as well. The reason women are more susceptible to TBL is still unclear. Tumor necrosis factor and interleukin-10 may play a role ${ }^{31} \cdot \mathrm{CD}_{4}+$ lymphocyte counts, endocrinal factors, socioeconomic factors, and cultural factors might also be involved in the gender difference. ${ }^{32}$ The most commonly involved lymph node of TBL in our study was mediastinal lymph nodes (32.4\%), followed by cervical lymph nodes (24.3\%), supraclavicular lymph nodes (21.6\%). Jia et al. found that the most commonly involved lymph node was cervical lymph nodes $86.7 \%$, followed by axillary lymph nodes $6.0 \%$ and inguinal lymph nodes 3.6\%. ${ }^{10}$ The inconsistencies in this aspect may be largely associated with differences in patients characteristics of the healthcare facilities, where the hospital in our study receives more complicated and severe cases with swollen deep lymph nodes.

Our study has several limitations. This is a retrospective study and the sample size is relatively small, which may introduce bias that leads to either over or under-estimation of the final outcome. In addition, as the healthcare institution where study was conducted is the National Center for Difficult and Complicated Cases, patients in this study are generally severe or complicated cases, the results should be applied with caution when T-SPOT.TB being applied to hospitals with different patient characteristics. It is necessary to perform multi-centric large sample-sized studies to further evaluate the diagnostic value of T-SPOT.TB.

In summary, the study suggests that T-SPOT.TB may be considered as a simple, rapid, highly sensitive tool for TBL diagnosis with good negative predictive value.

\section{Conflict of interest statement}

All authors declared no conflict of interests.

\section{Article information}

Partial results of this study were presented by poster at IDWeek conference 2017, San Diego.

\section{REFERENCES}

1. World Health Organization. Global Tuberculosis Report 2018. http://www.who.int/tb/publications/global_report/en/.

2. Pehme L, Hollo V, Rahu M, et al. Tuberculosis during fundamental societal changes in Estonia with special reference to extrapulmonary manifestations. Chest 2005; 127(4):1289-95. doi:10.1016/S0012-3692(15)34479-2.

3. Liao $\mathrm{CH}$, Chou $\mathrm{CH}$, Lai CC, et al. Diagnostic performance of an enzyme-linked immunospot assay for interferon-gamma in extrapulmonary tuberculosis varies between different sites of disease. J Infect 2009; 59(6):402-8.

doi:10.1016/j.jinf.2009.10.001. 
4. Song $\mathrm{KH}$, Jeon JH, Park WB, et al. Usefulness of the whole-blood interferon-gamma release assay for diagnosis of extrapulmonary tuberculosis. Diagn MicrobiolInfect Dis 2009; 63(2): 182-7. doi:10.1016/j.diagmicrobio.2008.10.013.

5. Benjelloun A, Darouassi Y, Zakaria Y, et al. Lymph nodes tuberculosis: a retrospective study on clinical and therapeutic feature. Pan African Med J 2015; 20: 65. doi:10.11604/pamj.2015.20.65.5782.

6. Shin JA, Chang YS, Kim HJ, et al. Diagnostic utility of interferon-gamma release assay in extrapulmonary tuberculosis. Diagn Microbiol Infect Dis 2015; 1(82): 44-8. doi:10.1016/j.diagmicrobio.2015.02.002.

7. Kim $\mathrm{KH}$, Kim RB, Woo SH, et al. The efficacy of the interferon-y release assay for diagnosing cervical tuberculous lymphadenitis: a prospective controlled study. Laryngoscope 2016; 126:378-84. doi:10.1002/lary.25540.

8. Kim YK, Uh Y, Lee NS, et al. Whole-blood interferon-gamma release assay for diagnosis of tuberculous lymphadenitis. Tohoku J Exp Med 2011; 224(3): 189-93. doi:10.1620/tjem.224.189.

9. Kim JK, Ko JJ, Kim KC, et al. Comparison of the tuberculin skin test and the interferon-y release assay for the diagnosis of cervical tuberculous lymphadenitis. Korean J Urol 2013; 56(6): 354-8. doi:10.3342/kjorl-hns.2013.56.6.354.

10. Jia H, Pan L, Du B, et al. Diagnostic performance of interferon-y release assay for lymph node tuberculosis. Diagn Microbiol Infect Dis 2016; 85(1):56-60. doi:10.1016/j.diagmicrobio.2016.02.001.

11. Lai CC, Liao CH, Tan CK, et al. Diagnosis of peripheral tuberculous lymphadenitis by enzyme-linked immunospot assay for interferon-gamma. Am J Med 2009; 122(8):e3. doi:10.1016/j.amjmed.2009.02.011.

12. Cho OH, Park KH, Kim SM, et al. Diagnostic performance of T-SPOT.TB for extrapulmonary tuberculosis according to the site of infection. J Infect 2011;6(3):362-9. doi:10.1016/j.jinf.2011.06.010.

13. Lu X, Li WP, Xie YH, et al. Evaluation of interferon-gamma release technique in extra-pulmonary tuberculosis detection, Chin J Lung Dis 2016; 1(9):20-5. doi:10.3877/cma.j.issn.1674-6902.2016.01.006.

14. Diel R, Loddenkemper R, Nienhaus A. Evidence-based comparison of commercial interferon-gamma release assays for detecting active TB: a meta analysis. Chest 2010; 137(4): 952-68. doi:10.1378/chest.09-2350.

15. Sadatsafavi M, Shahidi N, Marra F, et al. A statistical method was used for the meta-analysis of tests for latent TB in the absence of a gold standard, combining random-effect and latent-class methods to estimate test accuracy. J Clin Epidemiol 2010; 63(3): 257-69. doi:10.1016/j.jclinepi.2009.04.008.

16. Cambau E, Drancourt M. Steps towards the discovery of Mycobacterium tuberculosis by Robert Koch, 1882. Clin Microbiol Infect 2014; 20(3):196-201. doi:10.1111/1469-0691.12555.

17. Haldar S, Bose M, Chakrabarti $\mathrm{P}$, et al. Improved laboratory diagnosis of tuberculosis-the Indian experience. Tuberculosis 2011; 91 (5): 414-26. doi:10.1016/j.tube.2011.06.003.

18. Verma JS, Dhavan I, Nair D, et al. Rapid culture diagnosis of tuberculous lymphadenitis from a tertiary care centre in an endemic nation: potential and pitfalls. Indian J Med Microbiol 2012; 30: 342-5. doi: 10.4103/0255-0857.99498.

19. Agarwal AK, Sethi A, Sethi D, et al. Tubercular cervical adenitis: clinicopathologic analysis of 180 cases. J Otolaryngol Head Neck Surg 2009; 38(5):521-5. doi:10.2310/7070.2009.080212. 
20. Sinha P, Prakash P, Patne SC, et al. Performance of nested multiplex PCR assay targeting MTP40 and IS6110 gene sequences for the diagnosis of tubercular lymphadenitis. J Microbiol 2017; 55(1):63-7. doi:10.1007/s12275017-6127-y.

21. Ikram A, Ahmed SA, Khan FA, et al. Rapid Mycobacterium tuberculosis DNA detection on fine needle aspirates from extra pulmonary lymph nodes. J Coll Physicians Surg Pak 2015; 25(6):417-21. doi: 06.2015/JCPSP.417421.

22. Mittal $\mathrm{P}$, Handa $\mathrm{U}$, Mohan $\mathrm{H}$, et al. Comparative evaluation of fine needle aspiration cytology, culture, and PCR in diagnosis of tuberculous lymphadenitis. Diagn Cytopathol 2011; 39(11): 822-6. doi:10.1002/dc.21472.

23. Gillissen A. Tuberculosis-epidemiology, diagnostics and therapy. MMW Forts chr Med 2016; 158(6):50-5. doi: $10.1007 /$ s15006-016-7650-1.

24. Neonakis IK, Spandidos DA, Petinaki E. Female genital tuberculosis: a review. Scand J Infect Dis 2011; 43:564-72. doi: $10.3109 / 00365548.2011 .568523$.

25. Jia HY, Pan LP, Liu F. Adjunctive diagnostic value of interferon-gamma release assay in tuberculosis lymphadenitis. Chin J Antituberculosis 2014; 36(6): 467-71. doi:10.3969/j.issn.1000-6621.2014.06.012.

26. Pan L, Jia H, Liu F, et al. Risk factors for false-negative T-SPOT.TB assay results in patients with pulmonary and extrapulmonary TB. J Infect 2015; 70(4): 367-80. doi:10.1016/j.jinf.2014.12.018.

27. Hang NT, Lien LT, Kobayashi N, et al. Analysis of factors lowering sensitivity of interferon-gamma release assay for tuberculosis. PLoS One 2011; 6:e23806. doi:10.1371/journal.pone.0023806.

28. Gao L, Lu W, Bai L, et al. Latent tuberculosis infection in rural China: baseline results of a population-based, multicentre, prospective cohort study. Lancet Infect Dis 2015; 15(3):310-19. doi:10.1016/S1473-3099(14)71085-0.

29. Liu XQ, Bian SN, Cheng XH, et al. Utility of T-cell interferon-y release assays for the diagnosis of female genital tuberculosis in a tertiary referral hospital in Beijing, China. Medicine 2016; 95 (44): e5200. doi: $10.1097 / \mathrm{md} .0000000000005200$.

30. Wang LC, Yu Y, Chen W, et al. Evaluation of the characteristics of the enzyme-linked immunospot assay for diagnosis of active tuberculosis in China. Clin Vaccine Immunol 2015; 22:510-5. doi:10.1128/CVI.00023-15.

31. Temple SE, Pham K, Glendenning P, et al. Endotoxin induced TNF and IL-10 mRNA production is higher in male than female donors: correlation with elevated expression of TLR4. Cell Immunol 2008; 251(2):69-71. doi:10.1016/j.cellimm.2008.04.013.

32. Chan YM, Noertjojo K, Chan SL, et al. Sex differences in tuberculosis in Hong Kong. Int J Tuberc Lung Dis 2002; 6(1):118. doi:10.1067/mhl.2002.120259. 